Themenheft Nr. 44: Datengetriebene Schule.

Forschungsperspektiven im Anschluss an den 27. Kongress der DGfE

Herausgegeben von Mandy Schiefner-Rohs, Sandra Hofhues und Andreas Breiter

\title{
Auf dem Weg zu digital souverän agierenden Schulen
}

\section{Erste Erfahrungen und Erkenntnisse eines entwicklungsorientierten Modellschulprojektes}

\author{
Franco Rau ${ }^{1}$ (D) und Anna Geritan ${ }^{2}$ \\ ${ }^{1}$ Universität Vechta \\ 2 Technische Universität Darmstadt
}

\begin{abstract}
Zusammenfassung
Ziel des Beitrages ist, das Konzept und erste Erkenntnisse eines entwicklungsorientierten Forschungsprojektes zur Begleitung von Modellschulen auf ihrem Weg hin zu digital souverän agierenden Schulen zu präsentieren. In der Betrachtung von zwei ausgewählten Problem- und Handlungsfeldern wird empirisch fundiert beschrieben, mit welchen Herausforderungen digital weniger fortgeschrittene Schulen im Kontext aktueller Digitalisierungsdiskurse unter Pandemiebedingungen konfrontiert sind und wie die Gestaltung von Schulentwicklungsprozessen in der konkreten schulischen Praxis unterstützt werden kann. Das erste Problemfeld widmet sich der Frage, wie ausgehend von einem technisch vorhandenen Tablet-Klassensatz Lehrpersonen für mobiles Lernen zur Sprachförderung sensibilisiert werden können. Das zweite Problemfeld skizziert Herausforderungen und Ansätze zur Etablierung digitaler Lernplattformen an einer integrierten Gesamtschule. Die Projekterfahrungen dokumentieren Möglichkeiten zur Erschliessung neuer Handlungsspielräume und schulpraktische Bedingungen, die als einschränkend erlebt werden. Begrenzte Handlungsmöglichkeiten der Schulen wurden insbesondere bei Prozessen der Datenverarbeitung sowie Fragen des Datenschutzes sichtbar.
\end{abstract}

The Way to Digitally Empowered Schools. First Insights Into a Design-BasedResearch Project

\footnotetext{
Abstract

The article presents the concept and preliminary findings of a design-based-research project. We assisted schools on their way to digitally empowered schools. An empiricallyinformed description is given of the challenges faced by digitally less advanced schools
} 
under pandemic conditions in examining two selected projects. The article also shows ways to support the design of school development processes in concrete school practice. The first project is dedicated to learning with mobile devices at a school with a focus on promoting speech therapy. The second project outlines the challenges while establishing learning management systems at a comprehensive school. The two projects demonstrate new opportunities for schools to take action. At the same time, restrictive framework conditions become visible. The schools' limited options for action were particularly evident in data processing and privacy issues.

\section{Wissenschaftliche Begleitung von Modellschulen}

Mit dem Strategiepapier «Bildung in einer digitalen Welt» hat die KMK (2017) auf bildungspolitischer Ebene Ziele für Schulen und Lehrpersonen in Deutschland gesetzt. Zur Erreichung dieser Ziele, beispielsweise der Förderung der «Kompetenzen in einer digitalen Welt», können die Bundesländer, so die Formulierung der KMK (2017, 19), «verschiedene Wege beschreiten». Passend zu der Metaphorik der Fortbewegung werden Hochschulen in der «Rolle eines wissenschaftlichen Begleiters» (KMK 2017, 51) beschrieben. Diese Begleitung soll sowohl die unternommenen Schritte und Massnahmen hinsichtlich ihrer Wirksamkeit in den Blick nehmen als auch Impulse zur Weiterentwicklung liefern.

Die wissenschaftliche Begleitung von Pilot- und Modellschulprojekten diente in den letzten Jahren häufig dem Ziel, die Implementierung spezifischer technischer Infrastruktur und die Umsetzung pädagogischer Konzepte zum Lehren und Lernen in einer digital geprägten Welt systematisch zu dokumentieren und zu evaluieren. Zur Einführung der digitalen Plattform «LOGINEO NRW» in Nordrhein-Westfalen wurden beispielsweise 20 Pilotschulen wissenschaftlich begleitet, um Erkenntnisse über die schulische Arbeit mit der Plattform zu gewinnen und neue Bedarfe aus Sicht der Schulen zu identifizieren (Gerick et al. 2019a). Die wissenschaftliche Begleitung der Projekte «Start in die nächste Generation» in Hamburg (Kammerl et al. 2016), «Mobiles Lernen in Hessen» (Tillmann und Antony 2018) sowie "Lernen mit digitalen Medien» in Schleswig-Holstein (Gerick et al. 2019b) untersuchte jeweils die übergeordnete Frage, inwiefern es mit den Projekten gelungen ist, das Lernen mit und über digitale Medien im Unterricht zu verbessern. Neben dieser Zielstellung haben die exemplarisch genannten Projekte die Gemeinsamkeit, dass die Rolle der wissenschaftlichen Begleitung auf die Evaluation der jeweils unterschiedlichen Massnahmen beschränkt blieb.

Der vorliegende Beitrag präsentiert das Konzept und erste (Zwischen-)Ergebnisse eines Modellschulprojektes, in dem eine Universität aktiv in die Gestaltung der schulischen Praxis involviert ist. Um Erkenntnisse über die jeweils schulspezifischen Probleme zu gewinnen, zur unmittelbaren Verbesserung der Praxis beizutragen und 
die induzierten Veränderungsprozesse wissenschaftlich zu dokumentieren, orientiert sich das Projekt an Ansätzen der gestaltungs- und entwicklungsorientierten Bildungsforschung (z. B. Tulodziecki et al. 2013, Reinmann und Sesink 2014). Das Darmstädter Modellschulprojekt verfolgt das Ziel, Schulen auf ihrem Weg zu digital souverän agierenden Schulen zu begleiten. Handlungspraktisch soll die Entwicklung konkreter Unterrichtskonzepte ebenso wie die Auseinandersetzung mit grundlegenden Prozessen der Kommunikation, Informationsverarbeitung und Zusammenarbeit innerhalb des Bildungsraums Schule im Kontext einer digital geprägten Welt fokussiert werden. Da Digitalisierungsprozesse aus technischer Perspektive die Voraussetzung für vielfältige Prozesse der Datenspeicherung, -übertragung und -verarbeitung sind (Herzig 2020, 35), gehören dazu auch Fragestellungen hinsichtlich potenzieller Zugänge zu und Formen von schulbezogenen digitalen Daten sowie zur schulischen Daten- und Medienbildung.

Der Beitrag fokussiert zwei ausgewählte schulische Problemfelder zur Etablierung digitaler Infrastruktur als Voraussetzung für digital gestützte Lehr- und Lernprozesse für Lehrpersonen und Lernende. Auf Basis der Projekterfahrungen und ihrer empirischen Untersuchung wird beschrieben, mit welchen Herausforderungen sich Lehrpersonen an digital weniger fortgeschrittenen Schulen konfrontiert sehen und wie sie bei der Gestaltung von Schulentwicklungsprozessen in der schulischen Praxis unterstützt werden können. Zur Kontextualisierung werden zunächst die konzeptionellen Überlegungen des Gesamtprojektes vorgestellt (Kap. 2). Das erste Problem- und Handlungsfeld widmet sich der Auseinandersetzung mit einem Klassensatz Tablets, um das Lernen mit mobilen Endgeräten an einer Schule mit dem Schwerpunkt Sprachheilpädagogik zu unterstützen (Kap. 3). Das zweite Problem- und Handlungsfeld skizziert die Etablierung digitaler Lernplattformen an einer integrierten Gesamtschule (Kap 4). Die zwei ausgewählten Praxisbeispiele dokumentieren an den Schulen neu erschlossene Handlungsmöglichkeiten sowie einschränkende Rahmenbedingungen, unter denen Lehrpersonen in der Schule handeln müssen. In der Handlungspraxis ergeben sich schliesslich Fragen zum Umgang mit schulbezogenen Daten.

\section{Konzept und Methodik des Darmstädter Modellschulprojektes}

Zusammen mit dem Schulamt der Stadt und der Technischen Universität Darmstadt wurde ein Konzept für das Schulentwicklungsprojekt entworfen. Ziel des Vorhabens ist es, «Darmstädter Modellschulen» auf ihrem Weg zu digital souverän agierenden Schulen forschend zu begleiten (Rau et al. 2021). Durch die spezifische Art der empirischen Forschung im Modus einer entwicklungsorientierten Bildungsforschung sollen praxisbezogene Konzepte in Zusammenarbeit entwickelt, in der je spezifischen 
Handlungspraxis der Schulen erprobt sowie hinsichtlich ihrer Veränderungspotenziale untersucht werden. Der zugehörige Forschungsrahmen, die Umsetzung sowie konzeptionelle Überlegungen werden im Folgenden skizziert.

\subsection{Entwicklungsorientierte Bildungsforschung als Forschungsrahmen}

In der deutschsprachigen medienpädagogischen Diskussion wird regelmässig gleichwohl mit unterschiedlicher Akzentuierung - für mehr praxisorientierte Forschung plädiert (Spanhel 2007; Petko 2011; Niesyto 2014). Neben existierenden Konzepten schulbezogener Forschungs- und Entwicklungsarbeit (Einsiedler 2011, Kattmann 2007, Altrichter und Posch 2007) wurden in den letzten Jahren gestaltungsund entwicklungsorientierte Ansätze medienpädagogischer Bildungsforschung diskutiert (Reinmann und Sesink 2014; Tulodziecki et al. 2014; Preußler et al. 2014). Ein zentrales Argument für gestaltungs- und entwicklungsorientierte Ansätze ist die Praxisrelevanz der Forschungsergebnisse und der Nutzen, welcher in Bildungsforschung häufig problematisiert wird (Rau 2020). Der entwicklungsorientierte Forschungsrahmen umfasst drei aufeinanderfolgende Forschungsphasen, die in einem iterativen Vorgehen mehrmals nacheinander durchlaufen werden können (Abb. 1).

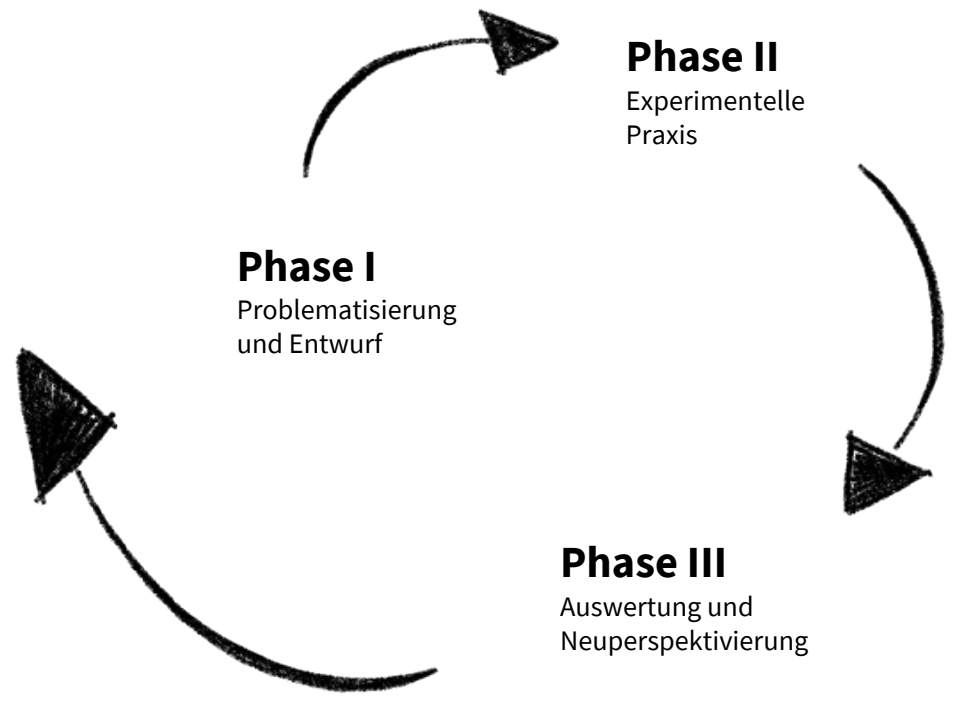

Abb. 1.: Vereinfachte Darstellung der Forschungsphasen einer entwicklungsorientierten Bildungsforschung (Rau, 2020).

Die erste Phase «Problematisierung und Entwurf» markiert für Sesink und Reinmann $(2015,71)$ den Beginn bzw. den Einstieg in ein Entwicklungsprojekt. In Abgrenzung zu anderen wissenschaftlichen Ansätzen geht es explizit nicht darum, ein Problem, welches aus der Perspektive der Wissenschaft in der Praxis gesehen wird, im jeweiligen Praxisfeld zu untersuchen. Vielmehr stellt sich für Forscherinnen und 
Forscher sowie Praktikerinnen und Praktiker in dieser Phase die Herausforderung, gemeinsam ein Problem, d. h. eine Diskrepanz zwischen der pädagogischen Praxis und den normativen Orientierungen zu erkennen. In Zusammenarbeit von Forschung und Praxis gilt es dann, einen ersten Entwurf zur Bearbeitung dieser Problematik zu erarbeiten. Die Form der Zusammenarbeit beschreiben Sesink und Reinmann (2015, 74) als «Entwicklungspartnerschaft», welche ein gemeinsames praktisches Engagement, eine «gemeinsame Verantwortung», für den Entwurf und dessen systematische Überprüfung umfasst.

In der zweiten Phase «Experimentelle Praxis» geht es nach Sesink und Reinmann $(2015,75)$ um die Durchführung des Entwicklungsprojektes: zum einen die Erprobung des in der ersten Phase entwickelten Entwurfs einer neuen, besseren Praxis (Sesink und Reinmann 2015, 75), zum anderen um die systematische empirische Untersuchung und Reflexion der «zu erprobenden Praxis» (Sesink und Reinmann 2015, 80). Entgegen einer Konzentration auf die Überprüfung von vorab zu erwartenden Ergebnissen zielt eine entwicklungsorientierte Bildungsforschung insbesondere auf das Neue und das Unvorhergesehene als Ergebnis eines neuen Anfangs ab (und nicht als Abweichung von kontrollierbaren Laborbedingungen).

Das Ergebnis der experimentellen Praxis beinhaltet Facetten, die es im Rahmen der dritten Phase «Auswertung und Neuperspektivierung» (Sesink und Reinmann $2015,80)$ zu interpretieren und zu diskutieren gilt. Ähnlich wie Tulodziecki et al. $(2013,2014)$ geht es Sesink und Reinmann $(2015,80)$ einerseits um die «empirische Feststellung, ob das eingetreten ist, was der Entwurf vorgesehen hatte». Anderseits ist eine forschungsmethodische Reflexion des empirischen Vorgehens und eine zukunftsgewandte Interpretation der Ergebnisse vorgesehen. Im Vergleich zu quantitativ empirischer Forschung erfolgt die zukunftsgewandte Interpretation der entwicklungsorientierten Bildungsforschung einer besonderen Intention. Metaphorisch ausgedrückt: «Ihre Intention ist nicht abschliessend, sondern aufschliessend, nicht feststellend, sondern 〈bewegend»» (Sesink und Reinmann 2015, 81). Mit Bezug auf Allert und Richter (2011) machen Sesink und Reinmann $(2015,80)$ darauf aufmerksam, dass die generierten Erkenntnisse zum Verstehen des Problems und zum Verstehen der Lösung beitragen. Im Sinne von Sesink und Reinmann (2015) bieten die Ergebnisse der ersten Iteration so einen Anknüpfungspunkt für die zweite Iteration.

\subsection{Konzeption und Aufbau des Projektes}

Die Idee des Projektes entstand in Zusammenarbeit von Forscherinnen und Forschern der TU Darmstadt, Vertreterinnen und Vertretern des Schulträgers sowie der Stadt Darmstadt. Um allgemeinbildende und berufliche Schulen in Darmstadt hinsichtlich vielfältiger Herausforderungen durch die Digitalisierung zu unterstützen und Erkenntnisse über notwendige Veränderungsprozesse zu gewinnen, wurde das 
Darmstädter Modellschulprojekt entworfen. Um die Vielfältigkeit der Schullandschaft abzubilden, wurden zur Durchführung des Projektes vom Schulträger drei interessierte Schulen ausgewählt, die sich hinsichtlich der Bildungsgänge, Anzahl der Lehrpersonen und Lernenden sowie der Ausstattung unterscheiden. Die ausgewählten Schulen werden für zwei Jahre im Sinne einer entwicklungsorientierten Bildungsforschung wissenschaftlich begleitet.

Mit dem Projektbeginn im Jahr 2019 wurde sichtbar, dass eine allgemeine Problemdefinition den jeweils spezifischen Bedürfnissen der Akteurinnen und Akteuren der Schulen sowie den jeweils vorhandenen Rahmenbedingungen nicht gerecht werden kann. Im Rahmen einer projektinternen Kick-Off-Veranstaltung mit den relevanten Stakeholdern des Gesamtprojektes wurden erstens die schulspezifischen Ausgangssituationen thematisiert. Zweitens wurde aus wissenschaftlicher Perspektive der Versuch unternommen, für Chancen und Herausforderungen aktueller Digitalisierungs- und Mediatisierungsprozesse zu sensibilisieren. Neben den Schulleitungen der Modellschulen und der Arbeitsgruppe «Allgemeine Pädagogik mit dem Schwerpunkt Medienpädagogik» der TU Darmstadt waren Vertreterinnen und Vertreter des Medienzentrums, des Schulamtes sowie der Stadtverwaltung anwesend. Zur Problematisierung wurde in Follow-Up-Treffen mit schulischen Arbeitsteams kurz-, mittelund langfristige Herausforderungen diskutiert und erste Entwürfe für gemeinsame Umsetzungsszenarien in kooperativer Weise erarbeitet. Das Ergebnis dieser Phase wurde in Form von Teilprojektsteckbriefen festgehalten. Ein Teilprojekt widmet sich jeweils einem schulspezifischen Problem und einem zugehörigen Entwicklungsziel. Die Bearbeitung dieses Teilprojektes erfolgte im Modus der entwicklungsorientierten Bildungsforschung (Abb. 2). Die zugehörigen Steckbriefe dokumentieren das Ausgangsproblem, Projekt- und Forschungsziele sowie geplante Aktivitäten. Die Entwicklung von Entwürfen umfasste je nach schulspezifischen Zielstellungen unterschiedliche Formate, z. B. Konzepte für Online-Seminare zum mobilen Lernen, Beratungsprozesse zur Auswahl digitaler Anwendungen zur Umsetzung von Kompetenzrastern und die Entwicklung eines Schutzkonzeptes (für den digitalen Raum). Pro Schule wurden im Projektzeitraum mehr als drei Teilprojekte bearbeitet. 


\section{Darmstädter Modellschulprojekt}
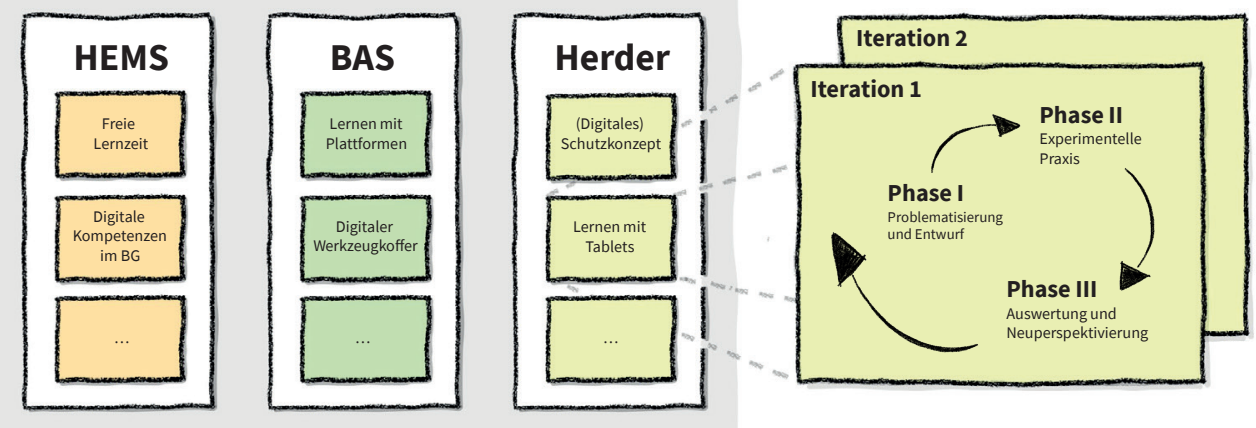

Abb. 2.: Schematische Visualisierung des Darmstädter Modellschulprojektes (eigene Darstellung).

Für die Phase der experimentellen Praxis wurde anknüpfend an die skizzierten Prinzipien von Sesink und Reinmann (2015) entschieden, qualitativ-empirische Forschungsansätze als primäre forschungsmethodische Herangehensweisen für das Gesamtprojekt zu verwenden. Zur methodischen Erfassung von Prozesserfahrungen erfolgte in Anlehnung an Sesink und Reinmann $(2015,82)$ z. B. eine Dokumentation der Projekterfahrungen durch Sitzungsprotokolle und durch individuelle Memos in Form von Forschungstagebüchern und Audioaufnahmen. Aufgrund der unterschiedlichen Schwerpunkte der einzelnen Teilprojekte wurden zudem gegenstandsspezifisch unterschiedliche Methoden der Datenerhebung und Auswertung gewählt. So wurden u. a. zur Evaluation von entwickelten Workshop-Angeboten Evaluationsbögen ausgearbeitet, welche sowohl offene als auch geschlossene Antworten beinhalteten. Zur Rekonstruktion der Perspektiven von Lehrpersonen im Hinblick auf die Zusammenarbeit in einer Arbeitsgruppe wurden hingegen episodische Interviews durchgeführt.

Mit den Ergebnissen der Erprobungen sollte die Frage beantwortet werden, inwiefern es gelungen ist, die im Vorfeld formulierten Ziele zu erreichen. Zudem sollten die Ergebnisse dazu beitragen, ein erweitertes Problemverständnis zu erzielen, um in Zusammenarbeit mit verschiedenen Personen der schulspezifischen Arbeitsgruppen die nächsten Schritte zur Weiterentwicklung der Praxis konzipieren zu können. Die zukunftsgewandte Diskussion der Ergebnisse erfolgte in den Teambesprechungen der wissenschaftlichen Begleitung der TU Darmstadt und im Austausch mit den Praxispartnerinnen und Praxispartnern der jeweiligen Schulen (z. B. Schulleitungen, Lehrpersonen). So führte die Diskussion und Interpretation der Ergebnisse zur Identifikation weiterer Entwicklungsziele und zur Fortsetzung der je konkreten Projekte. 


\subsection{Schulentwicklungsmodelle als Orientierungsrahmen}

In den vergangenen Jahren entstanden verschiedene praxisbezogene und wissenschaftliche Modelle zur systematischen Beschreibung und Reflexion der Zusammenhänge von Digitalisierungsaspekten und Schulentwicklungsprozessen. Als Strukturierungs- und Reflexionshilfe für das vorliegende Projekt wurde ein Modell gesucht, welches einerseits die verschiedenen Handlungsfelder komplexer Schulentwicklungsprozesse sichtbar machen kann und anderseits über eine wissenschaftliche Systematisierungsfunktion hinaus als hilfreich in der Zusammenarbeit mit Praktikerinnen und Praktikern erscheint. Während das SAMR-Modell (Puentedura 2006) Orientierungsangebote zur Unterrichtsentwicklung eröffnet und das TPACK- bzw. DPACK-Modell (z. B. Schmid und Petko 2020, Huwer et. al 2019) Strukturierungshilfen für Qualifikationsangebote von Lehrpersonen bietet, fokussieren entsprechende Modelle nur einzelne Dimensionen von Schulentwicklungsprozessen.

Ein relevantes Modell zur systematischen Einbeziehung digitaler Medien in der Schulentwicklung, welches über einzelne Handlungsfelder hinausgeht, wurde von Schulz-Zander (2001) vorgelegt. In diesem werden fünf Dimensionen der Schulentwicklung unterschieden: Neben der Unterrichts- und Personalentwicklung gehören für Schulz-Zander $(2001,272)$ die Kooperations-, Technologie- und die Organisationsentwicklung dazu. Aufbauend auf Schulz-Zander (2001) und weiteren Erkenntnissen präsentieren Lorenz und Bos (2017) ein analytisches Konzept zur systematischen Beschreibung von Qualitätsdimensionen schulischer Medienbildung hinsichtlich ihrer «relevanten Faktoren und Wirkmechanismen» (Lorenz und Bos 2017, 15). Auf einer Mehrebenenstruktur unterscheiden Lorenz und Bos (2017) zwischen Input-, Prozessund Outcomeebene des Schulsystems sowie externen Faktoren zur Beschreibung des Zusammenhangs von «Schulentwicklung und Schuleffektivität in Bezug auf digitale Medien» (Lorenz und Bos 2017, 13). Das Modell von Lorenz und Bos (2017) dient zur theoretischen Verortung der empirischen Befunde wissenschaftlicher Untersuchungen.

Ein stärker auf die Gestaltung von Praxis gerichtetes Modell formulierte Zylka $(2018,44)$ unter dem Titel «Vier-Wege-Modell der digitalen Schulentwicklung». In Erweiterung des Schulentwicklungsmodells von Rolff (2016) geht es Zylka (2018) darum, Digitalisierung - neben Personalentwicklung, Unterrichtsentwicklung und Organisationsentwicklung - als gleichwertiges Strukturmoment zu verstehen, welches wechselseitig mit den Aspekten verknüpft ist. Digitalisierung in diesem Verständnis ist mehr als eine «nette Ergänzung [...] zum regulären Betrieb» (Zylka 2018, 45) und nicht auf die Implementierung neuer Werkzeuge zur Verbesserung der Unterrichtsqualität zu reduzieren. Neben dieser Negativbestimmung bleibt bei Zylka (2018) weitgehend unbestimmt, welche Facetten unter dem Begriff Digitalisierung gefasst sind. 
Als wissenschaftlichen Orientierungsrahmen wurden für das Projekt «Darmstädter Modellschulen» die drei von Rolff (2016) vorgeschlagenen Handlungsfelder und Entwicklungsdimensionen der Personalentwicklung, Unterrichtsentwicklung und Organisationsentwicklung berücksichtigt. Zur expliziten Hervorhebung und Markierung technikbezogener Fragestellungen im Kontext aktueller Digitalisierungsprozesse wurde anknüpfend an die Argumentation von Zylka (2018) und Schulz-Zander (2001) ein weiteres Handlungsfeld ergänzt, welches als «Technologieentwicklung» bezeichnet wurde (Schulz-Zander 2001). Im Fokus dieses Handlungsfeldes stehen Fragen zur Bereitstellung, Wartung und Verwaltung der technischen Infrastruktur (z. B. Einrichtung von Tablets, Wartung von Serverstrukturen, Berücksichtigung des Datenschutzes). Die von Schulz-Zander (2001) markierte Dimension der Kooperationsentwicklung wurde als Querschnittsthema der vorherigen Entwicklungsdimensionen berücksichtigt. Die benannten Aspekte werden als sich wechselseitig bedingende Strukturmomente von Schulentwicklungsprozessen verstanden. Für die Entwicklung von praktischen Entwürfen wurde in diesem Modell die Chance gesehen, konkrete Handlungsfelder benennen zu können, die bei der Bearbeitung kurzfristiger Ziele im Fokus stehen (z. B. Qualifikationsangebote für Lehrpersonen zur Personalentwicklung). Zugleich können für langfristige Zielstellungen die jeweiligen Wechselbeziehungen zwischen verschiedenen Handlungsfeldern sichtbar gemacht werden. Die Etablierung einer Lernplattform auf technisch-organisatorische Massnahmen zu beschränken, blendet beispielsweise die notwendigen Kompetenzen von Lehrpersonen und Lernenden aus, um diese Plattform auch sinnvoll nutzen zu können.

Das skizzierte Modell diente im Projekt-Kick-Off und bei den anschliessenden Follow-Up-Treffen als Strukturierungs- und Reflexionshilfe, um auf die Komplexität von Schulentwicklungsprozessen aufmerksam und zu berücksichtigende Handlungsfelder sichtbar zu machen. Das eher abstrakte Modell lässt offen, wie sich die von unterschiedlichen Autorinnen und Autoren angenommen Abhängigkeiten der Handlungsfelder ausgestalten. Mit der Durchführung iterativer Forschungszyklen wird mit dem Projekt die Chance gesehen, diese Relationen der unterschiedlichen Handlungsfelder näher bestimmen zu können.

Als Zielbestimmung wurde im Projekt die Formulierung gewählt, Schulen auf dem Weg zu digital souverän agierenden Schulen zu begleiten. Diese Zielstellung bewegt sich in dem Spannungsfeld existierender bildungsorganisatorischer Leitbilder (z. B. KMK 2012, 2017), wissenschaftlicher Erkenntnisse zu Möglichkeiten und Herausforderungen für die Schule (z. B. Moser 2010, Döbeli Honegger 2016, Thumel et al. 2020) und den Perspektiven der Personen an den jeweiligen Schulen. Um im Kontext aktueller Digitalisierungsprozesse Lernende angemessen auf die Zukunft vorbereiten zu können, braucht es Schulen - Lehrpersonen, Verwaltungspersonal, Schülerinnen und Schüler -, die (a) die mit der Digitalisierung verbundenen Herausforderungen begreifen und (b) gegenstandsbezogene Urteils- und Handlungsfähigkeit 
zum Einsatz digitaler Technologien für menschliche Lern- und Arbeitsprozesse entwickeln. Der Grad an digitaler Souveränität ist aus unserer Perspektive jedoch nicht allein auf Wissen und Fähigkeiten der jeweils handelnden Personen zu beschränken, sondern wird durch «den von außen eingeräumten Grad der Möglichkeiten, Verfügung über die Bedingungen des eigenen Handelns [...] gewinnen zu können» (Müller 2019, 146), gerahmt. Wird das Konzept der «Lernenden-Souveränität» (Müller 2019, 149) auf Lehrpersonen und Einzelschulen übertragen, kann markiert werden, dass Bildung in einer digital vernetzten Welt auch durch die umgebenden Bedingungen der Schulentwicklung beeinflusst wird (z. B. Infrastruktur- und Fortbildungsangebote). In vergleichender Betrachtung der vielfältigen Teilprojekte werden diese Relationen für die innere und äussere Schulentwicklung im Gesamtprojekt in den Blick genommen und können in den folgenden Fällen exemplarisch veranschaulicht werden.

\section{Vom Tablet-Klassensatz zum mobilen Lernen zur Sprachförderung}

Im März 2020 wurde an einer Schule mit dem Schwerpunkt Sprachheilpädagogik in Zusammenarbeit mit der Schulleitung und der wissenschaftlichen Begleitung ein Projekt zum Lernen mit mobilen Endgeräten gestartet. Der Ausgangspunkt des Teilprojektes war die vorhandene technische Infrastruktur eines Klassensatzes Tablets an der Schule, welche bisher nicht systematisch in den Unterricht eingebunden wurden. Die folgenden Projekterfahrungen eröffnen einen Einblick über bestehende Herausforderungen zur Einbindung der technischen Geräte und erste Erkenntnisse, wie es in zwei Iterationen gelungen ist, durch das Angebot von Online-Workshops und die Gründung einer Arbeitsgruppe Beiträge zur Personalentwicklung von Lehrpersonen zu leisten.

\subsection{Unterstützungsbedarf zur Entwicklung von Mediennutzungskompetenzen}

Bei der ersten Problematisierung und dem gemeinsamen Entwurf durch das schulische Arbeitsteam - bestehend aus Vertreterinnen und Vertreter der Schule und der TU Darmstadt - zeigte sich, dass die Schule seit dem Jahr 2019 über einen Klassensatz an Tablets zur Gestaltung von mediengestützten Unterrichtsarrangements verfügt. Zugleich waren bis zu diesem Zeitpunkt nur wenige Lehrpersonen mit den Möglichkeiten von Tablets vertraut. Neben mangelndem Wissen zur sachgerechten Nutzung sowie zum mediendidaktischen Einsatz von Tablets für Unterrichtszwecke zeigten sich Entwicklungspotenziale auf der Ebene der technischen Wartung der Geräte und angemessener Unterstützungsstrukturen. Zum Umgang mit dieser Situation wurde im Kontext der pandemiebedingten Schulschliessungen von der Schulleitung und der wissenschaftlichen Begleitung die Idee entwickelt, Qualifizierungs- und Erfahrungsangebote zu schaffen. Das Ziel der Angebote war es, Lehrpersonen dabei 
zu unterstützen, die vorhandenen Tablets sachgerecht nutzen und perspektivisch eigene Unterrichtsszenarien zum Lernen mit Tablets entwickeln zu können. Zur Umsetzung der Ziele wurde ein digitales Workshopangebot von der wissenschaftlichen Begleitung in Zusammenarbeit mit beratenden Lehrpersonen entwickelt. Das Angebot sollte einen exemplarischen Einblick in unterschiedliche Szenarien zum Lernen mit Tablets eröffnen und die begleitete Erprobung dafür notwendiger Standardapplikationen ermöglichen. Ein Beispiel war die Erstellung eines mathematischen AudioPodcasts in der Primarstufe (Klose und Schreiber o. J.). Darauf aufbauend sollten die Lehrpersonen die Gelegenheit erhalten, die jeweiligen Applikationen in digitalen Gruppenräumen mit ihren Tablets zu erproben und beratend unterstützt zu werden.

Die Realisierung der Workshopangebote erfolgte in Form von zwei Onlineangeboten im April 2020. Auf Basis einer Evaluation der Online-Workshops erfolgte eine Bewertung der Qualität der Angebote, an der sich zehn von 14 Personen beteiligten. In einem funktionalen Verständnis beschreibt die Qualität das Ausmass, mit welchem die Fortbildung aus der Perspektive der Teilnehmenden die formulierte Zielstellung erfüllte (Harvey und Green 2000). Zur Einschätzung wurden die Lehrpersonen gebeten, Aussagen zum Online-Workshop auf einer fünfstufigen Likert-Skala zu bewerten $(1=$ «Stimme zu» bis 5 = «Stimme nicht zu»). Die Ergebnisse der Evaluation deuten darauf hin, dass die durchgeführten Online-Workshops aus der Perspektive der Lehrpersonen geeignet waren, um die angestrebten Ziele zu erreichen. Im Mittel stimmten alle Teilnehmer und Teilnehmerinnen den Aussagen zu, Anwendungen kennengelernt $(M=1,1)$ sowie Beispiele und Möglichkeiten zur Nutzung von Tablets aufgezeigt bekommen zu haben $(M=1,2)$. Zudem stimmten die Lehrpersonen den Aussagen eher zu, dass die Workshops Möglichkeiten eröffnet haben, konkrete Anwendungen zu erproben $(M=1,6)$, und sie fühlten sich durch den Workshop in der Lage, Tablets für eigene Unterrichtszwecke verwenden zu können ( $M=2)$. Zudem regte der OnlineWorkshop die Teilnehmenden zu einer weiteren Auseinandersetzung mit Tablets an $(M=1,3)$. Die Auswertung zeigt zudem, dass die Mehrheit der Teilnehmenden mit der fachlichen Betreuung durch die Workshop-Leitung zufrieden war und die technische Umsetzung des Online-Workshops als sehr positiv wahrgenommen hat.

Die Workshopangebote ermöglichten es, mit den vorhandenen Geräte ein Viertel des Kollegiums zu erreichen und Möglichkeiten zur anwendungsbezogenen Nutzung von Tablets zu erproben. Die Konzeption der Workshops und die Wahrnehmung der Angebote durch die Lehrpersonen kann als Beitrag zur Personalentwicklung der Schule verstanden werden. Dieser erste Beitrag zur Personalentwicklung konnte vor allem deshalb geleistet werden, weil die Lehrpersonen in diesem Zeitraum keinen verpflichtenden Distanzunterricht hatten und die Zeit für die individuelle Weiterbildung nutzten. In den Evaluationsergebnissen und in der Nachbesprechung der Online-Workshops mit den beteiligten Personen wurde mehrfach das Interesse geäussert, eine produktive Auseinandersetzung mit den Möglichkeiten von Tablets zur Unterrichtsentwicklung fortzuführen. 


\subsection{Begründete Auswahl von Apps als Chance und Herausforderung}

Aufbauend auf den Erfahrungen und der Evaluation der Workshops wurden in einer zweiten Iteration weitere Gespräche geführt, wie Tablets für den Unterricht nutzbar gemacht werden können, um dem spezifischen Förderschwerpunkt der Schule gerecht zu werden. Für den Förderschwerpunkt «Sprachheilpädagogik» kann einerseits auf bestehende App-Empfehlungen zurückgegriffen werden (Reber und Kaiser-Mantel 2020). Anderseits zeigte sich in der Phase der Problematisierung, dass bestehende Listen mit über $100 \mathrm{App}$-Empfehlungen für die Lehrpersonen nur wenig Orientierung boten. Zum Umgang mit der Frage, welche Apps für die Unterrichtsgestaltung zur Sprachförderung sinnvoll erscheinen und entsprechend über die Tablets bereitgestellt werden sollten, wurde die Idee entwickelt, eine Arbeitsgruppe zu gründen. Ziel der Arbeitsgruppe war es, App-Steckbriefe zu entwickeln, die eine Bewertung von Apps aus pädagogischer Perspektive eröffnen. Die App-Steckbriefe sollten als Orientierungshilfe für das Kollegium sowie als Entscheidungshilfe dienen, welche Apps auf den Geräten zu installieren sind.

Zur Realisierung der Zielstellung konnte im Sinne einer experimentellen Erprobung im Mai 2020 eine Arbeitsgruppe aus drei interessierten Lehrpersonen und einer studentischen Mitarbeiterin gegründet werden. Mit dem Fokus auf Sprachförderung entschied sich die Arbeitsgruppe das von Reber und Wildegger-Lack (2020) vorgeschlagene «Bewertungsnetz zur sinnvollen Auswahl realer und digitaler Medien» zu erproben. Nach den ersten Treffen der App-AG ist es gelungen, sich über den Umgang mit den jeweiligen Kriterien zu verständigen und das Bewertungsnetz für die eigene Arbeit anzupassen. Die Arbeit der App-AG umfasste in monatlichen Treffen (1.) die Auswahl von Apps zur Erprobung, (2.) die technische Einrichtung der Geräte und Installation der Apps, (3.) die Diskussion der zu bewertenden Apps sowie (4.) die Aufbereitung der Ergebnisse in Form von Steckbriefen. Im Zeitraum von Mai bis Dezember 2020 wurden in zehn Treffen insgesamt 62 verschiedene Apps ausgewählt und diskutiert. 38 Applikationen wurden von allen Personen der Arbeitsgruppe in Vorbereitung auf die Online-Treffen erprobt und kriteriengeleitet eingeschätzt. 20 Apps wurden hinsichtlich der Kriterien als sinnvoll eingestuft und in Form von Steckbriefen mit einem dem modifizierten Bewertungsnetz (Abb. 3) dokumentiert (Kosubski 2021). Die Arbeit der AG wurde in der Gesamtkonferenz vorgestellt und die App-Steckbriefe wurden dem Kollegium zur Verfügung gestellt. 


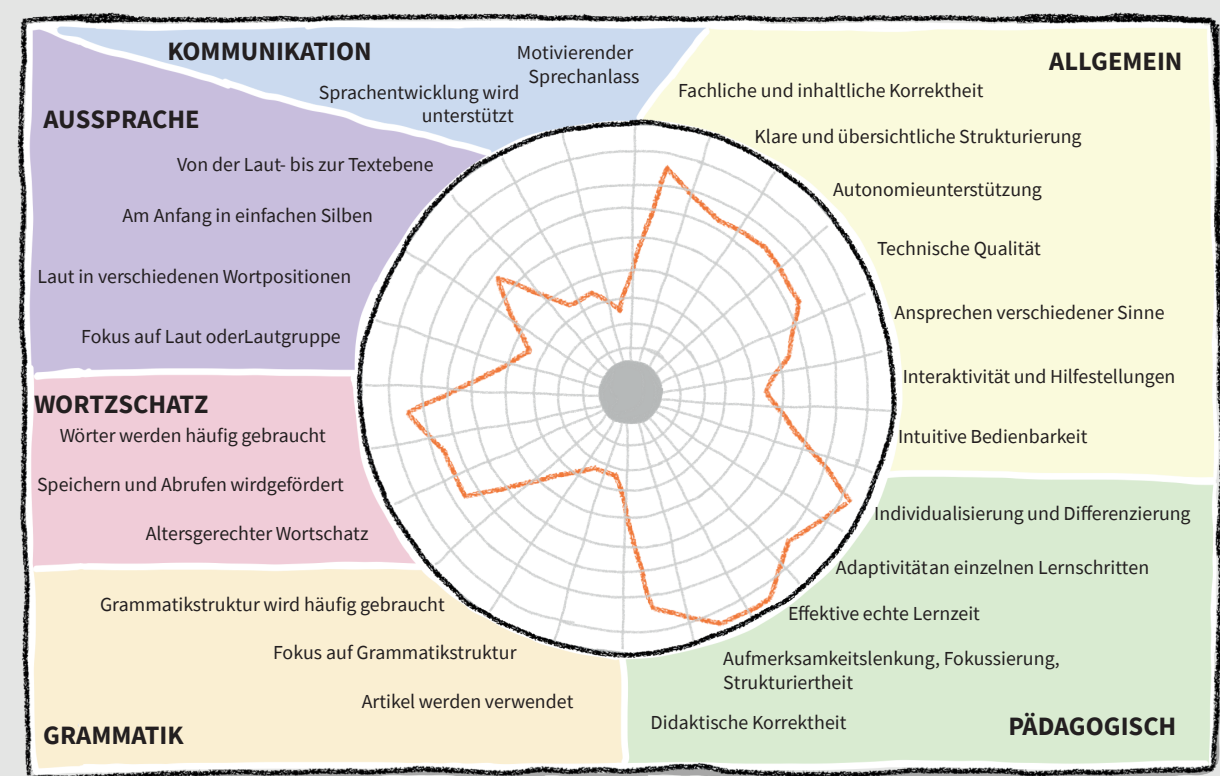

Abb. 1.: Bewertung der App «Zabulo» durch die Lehrpersonen im modifizierten Bewertungsnetz nach Reber und Wildegger-Lack (2020).

In Ergänzung zur pädagogischen Einschätzung und zur Einschätzung der Potenziale zur Sprachförderung wurde in den ersten Treffen des Jahres 2021 auch das Thema Datenschutz als Kriterium zur Bewertung der Apps diskutiert. Während die Lehrpersonen mit den Kriterien des Bewertungsrasters von Reber und WildeggerLack (2020) keine Probleme zum Ausdruck brachten und sich u. a. aufgrund ihres sonderpädagogischen Studiums als Expertinnen und Experten verstanden, waren die Lehrpersonen bei datenschutzbezogenen Fragen unsicher. Wenngleich Datenschutzfragen als relevantes Themenfeld erachtet wurden, wurde eine kriteriengeleitete Auseinandersetzung mit den AGB einer App von Lehrpersonen mitunter als den Fortschritt «bremsend» beschrieben (Kosubski 2021).

Zur wissenschaftlichen Dokumentation und Analyse wurden neben den dokumentierten Sitzungsprotokollen episodische Interviews mit den beteiligten Lehrpersonen im Januar 2021 durchgeführt (Kosubski 2021). Die ersten Ergebnisse einer qualitativen Analyse bieten Hinweise darauf, dass die Beteiligung an der App-AG einen Rahmen eröffnete, in dem sich Lehrpersonen kontinuierlich mit anwendungsbezogenen sowie didaktischen Fragestellungen zur Nutzung von Apps auseinandersetzen konnten. Aus der Perspektive der Lehrpersonen ermöglichte diese Auseinandersetzung u. a. die Weiterentwicklung eigener Medienkompetenzen und die Erweiterung von Vorstellungen zur Nutzung digitaler Anwendungen im Unterricht. Zudem 
berichten die Lehrpersonen von der Erfahrung, dass sie innerhalb des Kollegiums als die Expertinnen und Experten für Tablets wahrgenommen und zunehmend auf Tablets bezogene Fragen an sie gestellt werden.

Das aktuell noch laufende Projekt der App-AG skizziert erste Möglichkeiten, wie praktische Herausforderungen zur Implementierung der vorhandenen Infrastruktur in Form von Tablets zur Sprachförderung im Unterricht bearbeitet werden können. Dies betrifft die begründete Auswahl vorhandener Apps sowie Orientierungshilfen für Lehrpersonen, um sich mit der jeweiligen Infrastruktur vertraut zu machen.

\subsection{Diskussion}

Das vorgestellte Projekt mit den zwei skizzierten Iterationen eröffnet in diesem Beitrag einen genaueren Einblick darin, wie eine Schule vorgehen kann, um die vorhandene technische Infrastruktur vor Ort schrittweise für die spezifische Zielsetzung der Schule (z. B. zur Sprachförderung) nutzbar zu machen. Mit den gemeinsamen Aktivitäten zur Bewertung von Apps ist es auch gelungen, einen Beitrag zur Personalentwicklung zu leisten (Kosubski 2021). Dies äusserte sich beispielsweise hinsichtlich datenschutzbezogener Fragestellungen zur Verwendung ausgewählter Apps. So nahm die Sensibilität für eine szenarienbezogene Einschätzung der Datenschutzbestimmungen der jeweiligen Apps bei den beteiligten Lehrpersonen zu.

Zugleich wurden souveränitätseinschränkende Bedingungen sichtbar, welche sich auf technische Fragen der Datenverarbeitung sowie auf Fragen des Datenschutzes zurückführen lassen. Beispielsweise ist sich die Schulleitung einerseits bewusst, dass die Nutzung eines Mobile Device Management (MDM-)Systems die technische Wartung der Tablets deutlich vereinfachen könnte. Anderseits wird vom zuständigen Schulamt bisher nicht die Option eröffnet, ein zentrales MDM-System nutzen zu können. Stattdessen heisst es in einer im November 2020 verfassten Rundverfügung des Schulamts Darmstadt (2020) zur Einrichtung von Tablets für Schülerinnen und Schüler u. a. «Nehmen Sie bitte Abstand davon sich ein eigenes MDM System zu beschaffen. Ein zentrales System ist bereits wie eingangs erwähnt, in Vorbereitung». Dass sich die Etablierung des MDM-Systems bereits seit Projektbeginn im Januar 2020 in Vorbereitung befindet, ist für die Schule zum einen bedauerlich. Zum anderen steht diese Beobachtung exemplarisch für eine grundlegende Schwierigkeit verschiedener Landkreise. Die Bearbeitungsdauer des skizzierten Problems ist u. a. von der Klärung datenschutzbezogener Fragestellungen abhängig. Diese Klärung muss in Zusammenarbeit mit den jeweiligen Datenschutzbeauftragen der involvierten Institutionen erfolgen. Dabei ist nicht der Anspruch an datenschutzkonforme Softwarelösungen als souveränitätseinschränkende Bedingung zu sehen, sondern vielmehr die fehlende Kapazität der beteiligten Institutionen zur Klärung dieser zunehmend komplexen Fragestellungen. Schulentwicklungsprozesse können hinsichtlich der verschiedenen 
Handlungsfelder in diesem Zusammenhang nicht nur durch Personen der jeweiligen Einzelschule gestaltet werden, sondern sind zugleich abhängig von Personen der äusseren Schulentwicklung, z. B. vom Schulträger.

\section{Etablierung digitaler Lernplattformen unter Pandemiebedingungen}

Mit Beginn der Schulschliessungen im März 2020 wurde an einer integrierten Gesamtschule in Zusammenarbeit mit der Schulleitung und der wissenschaftlichen Begleitung ein Projekt zur Etablierung digitaler Lernplattformen gestartet. Ein Ausgangspunkt dieses Projekts war der entstandene Bedarf, mit den Schülerinnen und Schülern in digitalen Formaten in Kontakt zu bleiben sowie den Lern- und Unterrichtsbetrieb aufrecht zu erhalten. Die folgenden Projekterfahrungen eröffnen einen Einblick in die spezifischen Herausforderungen und liefern erste Erkenntnisse zur unterstützenden Begleitung. In zwei Iterationen wird beschrieben, wie vielschichtig sich dieser Prozess gestaltet und inwiefern Beiträge zur Personal- und Organisationsentwicklung sowie zur Technologieentwicklung geleistet werden konnten. Dabei dienen Sitzungsprotokolle sowie Forschungsmemos als empirische Datengrundlage. Souveränitätsermöglichende sowie -einschränkende Aspekte des Projektes werden in der Diskussion zusammengefasst.

\subsection{Die Wahl einer Lernplattform als Qualifizierungsanlass und Herausforderung} Lern- und Kommunikationsplattformen sind aus Sicht der KMK $(2017,40)$ «integraler Bestandteil schulischer Infrastrukturen» und markieren einen zentralen «Eckpfeiler» für eine «Bildung in einer digitalen Welt». Zur Verfolgung dieses Leitbildes, welches insbesondere im Kontext der pandemiebedingten Schulschliessungen (Pressestelle Hessische Staatskanzlei 2020) enorm an Bedeutung gewann, wurde von einer im Modellschulprojekt beteiligten Gesamtschule die Etablierung einer schulischen Lern- und Kommunikationsplattform als priorisiertes Entwicklungsziel formuliert. In der Phase der Problematisierung und des Entwurfes wurde in einer Arbeitsgruppe - bestehend aus Lehrpersonen, der Schulleitung und Personen der TU Darmstadt Moodle als eingebundene Plattform des Schulportals LANiS in Hessen und Microsoft Teams (MS Teams) als Anwendung von Microsoft 365 als die zwei bevorzugten Plattformen diskutiert. Zur Einbindung des Kollegiums bei der Auswahl wurde im Sinne einer partizipativen Entscheidungsfindung das Ziel verfolgt, interessierten Lehrpersonen erste Erfahrungen mit den jeweiligen Onlineanwendungen zu ermöglichen, um sich begründet für eine Plattform entscheiden zu können. Zur Erreichung wurden im Rahmen der Arbeitsgruppe verschiedene Aktivitäten geplant. Der IT-Beauftragte der Schule und Lehrpersonen fokussierten die technische Einrichtung der jeweiligen Plattformen und die Schaffung von Zugängen für interessierte Personen. Zur 
Sensibilisierung von Lehrpersonen wurden zudem Online-Workshopangebote von der wissenschaftlichen Begleitung entwickelt, um Berührungsängste abzubauen, Orientierung zu bieten und eine erste Einarbeitung in die Benutzeroberfläche in die Wege zu leiten. Damit sollte ein erster Beitrag geleistet werden, um Lehrpersonen perspektivisch in die Lage zu versetzen, sich an der partizipativen Entscheidungsfindung für eine Plattform begründet beteiligen zu können.

Um den Lehrpersonen eine erste Begegnung und einen niedrigschwelligen Zugang zu den ausgewählten Plattformen zu ermöglichen, wurden in der experimentellen Praxis mehrere Online-Workshops für die zwei unterschiedlichen Plattformen entwickelt. Es wurden ein Überblick über die ausgewählten Plattformen geboten und verschiedene Aspekte vergleichend gegenübergestellt. Beispielkurse innerhalb der Plattformen dienten, auch über die Workshops hinaus, zur Visualisierung der technischen Möglichkeiten sowie zum experimentellen Erkunden und Ausprobieren. Daneben wurden den Lehrpersonen Selbstlernmaterialien (schriftliche Anleitungen, Videotutorials etc.) zur Aneignung von MS Teams und Moodle zur Verfügung gestellt. Nach Abschluss der Fortbildungsreihe bot eine über fünf Monate regelmässig stattfindende Sprechstunde dem Kollegium eine weitere Möglichkeit der Unterstützung in Form von Beratung bei aufkommenden Fragen im Umgang mit den beiden Plattformen.

Zur wissenschaftlichen Analyse der entwickelten Workshop-Angebote wurden Daten aus der Perspektive der teilnehmen Personen in Form einer Online-Evaluation erfasst. Im Fokus stand die Frage, inwiefern es mit den entwickelten Weiterbildungsangebot gelungen ist, Lehrpersonen die Möglichkeit zu eröffnen, die Plattformen kennenlernen und erproben zu können. Zur Einschätzung wurden die Lehrpersonen gebeten, Aussagen auf einer fünfstufigen Likert-Skala zu bewerten («Stimme zu» (1) bis «Stimme nicht zu» (5)). In offenen Antworten konnten Kritik und Verbesserungsvorschläge zum Ausdruck gebracht werden. Von den 43 Teilnehmerinnen und Teilnehmern der Workshops beteiligten sich 16 Personen an der Evaluation. Die Ergebnisse der Evaluationen zeigen, dass die Fortbildungen in Form von Online-Workshops geeignet waren, die angestrebten Ziele zur Unterstützung der Lehrpersonen zu erreichen. So stimmten die Lehrpersonen, die an der Evaluation teilnahmen, der Aussage zu, dass sie durch den Online-Workshop die Chance hatten, die jeweilige Plattform kennenzulernen ( $M=1,3)$, und zur weiteren Auseinandersetzung mit Moodle bzw. MS Teams angeregt wurden $(M=1,2)$. Zudem fühlten sich die Lehrpersonen über die Möglichkeiten der jeweiligen Plattformen informiert $(M=1,5)$ und haben Wege erfahren, ihren Unterricht durch die Verwendung der jeweiligen Plattform unterstützen zu können $(M=1,5)$. Die Auswertung zeigt zudem, dass die Teilnehmenden mit der technischen Umsetzung sowie mit der fachlichen Betreuung durch die WorkshopLeiterinnen zufrieden waren. 
Mit den vorgestellten Projektaktivitäten ist es gelungen, die Lehrpersonen bei der Erprobung der jeweiligen Plattform aktiv zu unterstützen und Sensibilisierungsangebote zur Personalentwicklung des Kollegiums zu leisten. So nahm etwas weniger als die Hälfte des Kollegiums der Modellschule an den freiwilligen Workshops teil.

In der Auswertung und Neuperspektivierung der Evaluationsergebnisse und der gesammelten Projekterfahrungen wurden zwei zentrale Herausforderungen sichtbar, welche die geplante partizipative Entscheidungsfindung für eine Plattform durch das Kollegium verhinderten. Die durch das Schulportal des Landes Hessen nutzbare Moodle-Instanz für die Schule eröffnete einerseits eine datenschutzkonforme Nutzung. Andererseits war der Zugriff auf diese Plattform während der pandemiebedingten Schulschliessung nur eingeschränkt möglich. Diese Einschränkung entstand insbesondere durch Überlastung der entsprechenden Server, was sich u. a. in Ladezeiten einer Moodle-Seite von mehreren Minuten äusserte. So berichteten Lehrpersonen davon, dass eine Exploration der Plattform in der Erprobungsphase während der Schulschliessung nur in der Nacht möglich war. Aus der Perspektive der Lehrpersonen zeigten sich bei MS Teams im Vergleich keine entsprechenden Probleme in der Performanz. Jedoch existierten im Frühjahr 2020 Unklarheiten und Bedenken bezüglich einer datenschutzkonformen Nutzung von Microsoft 365 im Bildungsraum Schule. Die Kritik bezieht sich auf datenschutzrechtliche Problematiken und kommerzielle Interessen des Microsoft Konzerns (Fischer et al. 2020). Weder von Seiten der Stadt Darmstadt noch von Seiten des Landes Hessen lag zu diesem Zeitpunkt eine klärende Stellungnahme zur Nutzung von MS Teams im Kontext von Schule und Unterricht vor. Auf bundesweiter Ebene sorgen zum Teil widersprechende Regelungen für weitere Irritation. Diese zum Teil kontroversen (öffentlichen) Debatten verhinderten die geplante Entscheidungsfindung.

\subsection{Technisch-organisatorische Massnahmen zur Etablierung in der Schule}

Zum Umgang mit diesem Spannungsfeld wurde in einer zweiten Iteration die vorläufige Entscheidung getroffen, (vorübergehend) beide Plattformen verwenden zu wollen. Die Nutzung beider Angebote wurde von der schulischen Arbeitsgruppe als Chance gesehen, die Handlungsfähigkeit der Schule im Kontext der Pandemie zu sichern. Zudem erschien die «zweigleisiges Lösung den unterschiedlichen Bedürfnissen der Lehrpersonen und den unterschiedlichen Kompetenzen der Schülerinnen und Schüler zu entsprechen. Zur Etablierung der Plattformen wurde in der Entwurfs- und Planungsphase entschieden, relevante technische und organisatorische Massnahmen zu identifizieren und die Durchführung vorzubereiten (siehe auch DSK 2018). Relevante Aufgaben umfassten die Erstellung eines Verzeichnisses von Verarbeitungstätigkeiten, die Inkenntnissetzung der Betroffenen über die Nutzung der digitalen Plattformen, das Einverständnis der Erziehungsberechtigten, die Verteilung von Rollen und 
Berechtigungen, die Erstellung von E-Mail-Adressen und Accounts, die Verwaltung von Zugriffsrechten, das Einpflegen verschiedener Bereiche in die Online-Plattformen (Klasse, Kurse, AGs etc.) sowie eine angeleitete Begleitung (Orientierung bei der Navigation auf der Benutzeroberfläche) der Schülerinnen und Schüler beim ersten Login und die individuelle Erstellung und Verwaltung von Passwörtern.

Die Realisierung der geplanten Aktivitäten erfolgte arbeitsteilig durch die Mitglieder der schulischen Arbeitsgruppe entsprechend ihrer Kompetenzen und Kapazitäten. Die Durchführung der geplanten Massnahmen im Sinne einer experimentellen Praxis und wissenschaftlichen Begleitung ermöglichte es, als herausfordernd erlebte Phänomene zu dokumentieren, wie sich u. a. hinsichtlich der Massnahme zur Erstellung und Verwaltung von Passwörtern für Schülerinnen und Schülern zeigt. Die Verwaltung von Passwörtern war nicht nur eine organisatorische Aufgabe, sondern zugleich ein exemplarischer Anlass für eine medienbezogene Kompetenzentwicklung sowohl auf Seiten der Schülerinnen und Schüler als auch auf Seiten der Lehrpersonen (z. B. Eggert et al. 2016). Die Nutzung digitaler Plattformen ist mit einem passwortgesicherten Zugang verbunden. Schülerinnen und Schüler stehen dadurch vor der Aufgabe, individuelle und sichere (d. h. komplexe und geheime) Passwörter zu vergeben. Bei der Verwendung mehrerer accountgebundener Dienste und Anwendungen müssen folglich diverse Zugangsdaten in Erinnerung gerufen werden. Basierend auf vorangegangenen Erfahrungen der Lehrpersonen stellt dies für viele Schülerinnen und Schüler, besonders niedriger Jahrgänge, eine Herausforderung dar. Die Praxis verdeutlichte dem Lehrpersonal, dass Zugangsprobleme keine Seltenheit sind und zudem viel Zeit und Raum im Unterrichtsgeschehen einnehmen können, woraus der Wunsch nach einer zentralisierten Lösung (entgegen der Geheimhaltungspflicht) für die Verwaltung von Passwörtern resultierte. Aus medienpädagogischer und datenschutzrechtlicher Sicht werden die Zugänge im Idealfall selbstgesteuert verwaltet, wobei sich beispielsweise bei der Vergabe von Passwörtern an einer Vorlage orientiert werden kann. Die Auseinandersetzung mit Fragen zum Thema Passwortverwaltung wird dadurch zu einem Anlass zur Auseinandersetzung mit den Themen Datenschutz, Medienkompetenzvermittlung und Praktikabilität in der Schulpraxis.

In der Auswertung und Neuperspektivierung der zweiten Iteration zeigte sich in Diskussionen der Arbeitsgruppe, dass sich die Etablierung der Lernplattformen weiterhin in einem dynamischen Prozess befindet. Während in der Arbeitsgruppe zunehmend der Wunsch geäussert wurde, in folgenden Iterationen verstärkt Möglichkeiten der Unterrichtsentwicklung mit digitalen Lernplattformen zu entwickeln, wurde in einer aktuellen Entscheidung des Hessischen Beauftragten für Datenschutz und Informationsfreiheit die Duldung von MS Teams aufgehoben (HBDI 2021). Entsprechend werden auch in den folgenden Iterationen Fragen der technischen Infrastruktur bzw. des Datenschutzes weiterhin zum Thema gemacht werden. 


\subsection{Diskussion}

Digitale Lernplattformen fungieren im Idealfall unterstützend und begleitend bei der Realisierung des Bildungs- und Erziehungsauftrages der Schule. Das Lehren und Lernen kann durch eine entsprechende Bereitstellung unterstützt und ergänzt werden. Wie sich bei den skizzierten Projekterfahrungen zeigt, ist die Etablierung eines entsprechenden Angebotes als umfangreicher Prozess zu verstehen, dessen Umsetzung auf mehreren Handlungs- und Entwicklungsebenen zu verorten ist. Insbesondere auf Basis der Protokolle der AG-Sitzungen und in den durchgeführten Gesprächen mit Lehrpersonen zeigt sich, dass sich aufgrund der etablierten technischen Infrastruktur neue Handlungsspielräume zur digitalen Interaktion mit Schülerinnen und Schülern potenziell eröffnen und auch aktiv genutzt werden. Durch die technisch zur Verfügung stehenden Handlungsmöglichkeiten hat sich der Grad an digitaler Souveränität insbesondere für Lehrpersonen erhöht. So nutzen und erarbeiten aktuell beispielsweise einzelne Lehrpersonen Blended Learning-Konzepte für ihren Fachunterricht.

Zugleich dokumentieren die im Rahmen des Projektes «Etablierung digitaler Plattformen» gemachten Erfahrungen, wie Fragen der Datenverarbeitung sowie des Datenschutzes souveränitätseinschränkend erlebt wurden und sich zugleich zumindest potenzielle Entwicklungsanlässe eröffneten. Obwohl Datenschutz und Performance einer Plattform nicht im Widerspruch zueinanderstehen müssen, erlebten Lehrpersonen die Erprobung der digitalen Plattformen mitunter so, als hätten sie die Wahl zwischen einer performanten aber nicht datenschutzkonformen Lösung oder einer datenschutzkonformen aber nicht performanten Alternative. Der von Lehrpersonen geäusserte Bedarf an angemessen Unterstützungsstrukturen erscheint in diesem erlebten Spannungsfeld nachvollziehbar. Zudem zeigten sich Herausforderungen durch die Komplexität des Themas «Datenschutz» und die fehlende Expertise bei Fragen technischer Datenverarbeitungsprozesse. Die möglichen Beratungsangebote zur Auseinandersetzung mit diesen Fragen im Projektkontext eröffneten zugleich Entwicklungsanlässe für Lehrpersonen sowie Schülerinnen und Schüler, die beispielsweise in medienpädagogischen Unterrichtseinheiten zum Thema Passwortsicherheit umgesetzt werden konnten.

\section{Fazit: Erste Schritte und Hürden auf dem Weg}

Mit dem Beitrag wurde das Ziel verfolgt, in einer ersten Annäherung aktuelle Problem- und Handlungsfelder zu beschreiben, denen digital weniger fortgeschrittene Schulen im Rahmen von Schulentwicklungsprozessen zur Bildung in einer digital geprägten Welt begegnen. Zudem wurden Ansätze zur Begleitung und Unterstützung entsprechender Schulentwicklungsprozesse in der Handlungspraxis auf Basis von Projekterfahrungen und -erkenntnissen vorgestellt. Es wurde gezeigt, welche Schritte in exemplarisch ausgewählten Teilprojekten unternommen wurden, um Beiträge 
zur Personal- und Organisationsentwicklung sowie zur Technologieentwicklung innerhalb von Schule zu leisten. Durch die Entwicklung von Online-Workshops ist es beispielsweise gelungen, Lehrpersonen neue Handlungsmöglichkeiten im Umgang mit Tablets und digitalen Lernplattformen zu eröffnen. Zudem zeigte sich, dass die Etablierung einer technischen Infrastruktur für die zwei Modellschulen durch weitere Institutionen gerahmt wird. Diese Rahmung - im Sinne des Grades der von aussen eingeräumten (bzw. nicht eingeräumten) Handlungsmöglichkeiten (Müller 2019) wurde insbesondere bei Prozessen der Datenverarbeitung sowie in Bezug auf Fragen des Datenschutzes sichtbar. Beispielsweise stellte sich sowohl bei der Etablierung von Schulplattformen sowie bei der Nutzung konkreter Apps die Frage, was mit den Daten der Schülerinnen und Schüler passiert, wo diese gespeichert, weitergeleitet oder verarbeitet werden. Die Auseinandersetzung mit diesen Fragen eröffnete in beiden Teilprojekten potenzielle Anlässe, um zu einer Sensibilisierung für Datenschutz im Kontext digitaler Lernumgebungen sowie für die datenbezogene Sorgfaltspflicht von Lehrpersonen bei der Konzeption digitaler Lehr- und Lernangebote beizutragen. Diese Anlässe konnten für individuelle sowie institutionelle Entwicklungsprozesse in unterschiedlichem Ausmass ausgeschöpft werden.

Wird das Konzept der digitalen Souveränität von Müller (2019) sowie von Müller et al. (2020) auf Schulen als einzeln zu betrachtende Handlungseinheiten übertragen, kann beschrieben werden, wie (pandemiebezogene) Schulentwicklungsprozesse von Einzelschulen durch die von aussen zu Verfügung gestellte Infrastruktur gerahmt werden. Dies betrifft insbesondere die Entscheidung für die bereitgestellte Infrastruktur durch den Schulträger bzw. das jeweilige Bundesland, welche gemäss KMK (2017) «über die Einzelschule hinaus getroffen werden [sollte], möglichst auf Schulträgerebene, idealerweise auf Landesebene» (KMK 2017, 40). Bildungsadministrative Rahmenbedingungen variieren jedoch teilweise erheblich zwischen verschiedenen Landkreisen und auch Bundesländern. Exemplarisch zeigt sich dies bezüglich Ausstattungsfragen und Fragen zur Technologieentwicklung in der unterschiedlichen Umsetzung des Digital-Pakts durch die einzelnen Bundesländer (Jude et al. 2020). Zudem werden Fragen zur Digitalisierung auch innerhalb eines Bundeslandes unterschiedlich bzw. teilweise widersprüchlich verhandelt. Exemplarisch dafür steht der Umgang mit dem Datenschutz in Baden-Württemberg, wie die BigBrother-Awards ausführlich dokumentieren (Fischer et al. 2020). Die vorgestellten Einzelfälle zeigen ebenfalls, dass die Schulen über die jeweilige Infrastruktur nicht eigenständig verfügen können. Digital weniger fortgeschrittene Schulen, also etwa solche ohne lokale und selbstverwaltete Serverstrukturen, sind abhängig von kommerziellen Anbietern bzw. dem Schulträger. Wie sich in der Handlungspraxis der skizzierten Fälle zeigte, wurde diese Rahmung aufgrund verschiedener Probleme hinsichtlich der Datenverarbeitung und des Datenschutzes in unterschiedlichen Kontexten eher beschränkend als ermöglichend erlebt. 
In vergleichender Betrachtung der von der KMK (2017) formulierten Leitbilder und Umsetzungsstrategien sowie der schulischen Praxis in den vorgestellten Einzelfällen lässt sich für das Jahr 2020 eine deutliche Diskrepanz erkennen. Hinsichtlich der Verarbeitung schulbezogener Daten formulierte die KMK (2017) beispielsweise, dass «leistungsfähige Infrastrukturen (sowohl der Länder und Schulträger als auch länderübergreifend) unter Wahrung der Datensicherheit, des Datenschutzes» (KMK 2017, 34) bereitzustellen sind und «die Sicherheit der hoch sensiblen Nutzer- und Nutzungsdaten und deren datenschutzkonforme Übermittlung gewährleistet werden [muss]» (KMK 2017, 35). In den im vorliegenden Beitrag präsentierten Projekten zeigte sich, dass die Schulen nur begrenzt auf eine entsprechende unterstützende Infrastruktur zurückgreifen konnten. Diese durch die Pandemie sichtbar gewordene Problematik kann dabei durchaus als Entwicklungschance begriffen werden. Dafür ist jedoch notwendig, dass digitale Infrastruktur nicht auf die Ausstattung von Endgeräten reduziert bleibt. Vielmehr sollte es darum gehen, die bereits im Strategiepapier formulierten Absichtserklärungen der KMK (2017) hinsichtlich weiterer Beratungs- und Unterstützungsangebote für Schulen, Schulträger und Bundesländer inklusive entsprechender personeller Ressourcen tatsächlich umzusetzen. Ansätze einer entwicklungsorientierten Bildungsforschung können in diesem Zusammenhang Möglichkeiten bieten, lokal und regional-vergleichend spezifische Problemfelder und Herausforderungen zu identifizieren und zu deren produktiven Bearbeitung beizutragen.

\section{Literatur}

Allert, Heidrun, und Christoph Richter. 2011. «Designentwicklung - Anregungen aus Designtheorie und Designforschung». In Lehrbuch für Lernen und Lehren mit Technologien, herausgegeben von Sandra Schön und Martin Ebner, 1-14. Graz: L3T. http://l3t.eu/homepage/ das-buch/ebook/kapitel/o/id/50/name/designentwicklung.

Altrichter, Herbert, und Peter Posch. 2007. Lehrerinnen und Lehrer erforschen ihren Unterricht: Unterrichtsentwicklung und Unterrichtsevaluation durch Aktionsforschung. 4., überarb. und erw. Aufl. Bad Heilbrunn: Klinkhardt.

Bos, Wilfried, und Ramona Lorenz. 2017. «Konzeption, Anlage und Durchführung des Länderindikators 2017». In Schule digital - der Länderindikator 2017 Schulische Medienbildung in der Sekundarstufe I mit besonderem Fokus auf MINT-Fächer im Bundesländervergleich und Trends von 2015 bis 2017, herausgegeben von Ramona Lorenz, Wilfried Bos, Manuela Endberg, Birgit Eickelmann, Silke Grafe, und Jan Vahrenhold, 36-48. Münster: Waxmann. https://doi.org/10.25656/01:15656.

Döbeli Honegger, Beat. 2016. Mehr als 0 und 1. Schule in einer digitalisierten Welt. Bern: hep Verlag. 
DSK Datenschutzkonferenz. 2018. «Orientierungshilfe der Datenschutzaufsichtsbehörden für Online-Lernplattformen im Schulunterricht». https://www.datenschutzkonferenz-online. de/media/oh/20180426_oh_online_lernplattformen.pdf.

Einsiedler, Wolfgang. 2011. «Was ist Didaktische Entwicklungsforschung?» In Unterrichtsentwicklung und didaktische Entwicklungsforschung, herausgegeben von Wolfgang Einsiedler, 41-70. Bad Heilbrunn: Klinkhardt.

Eggert, Karin, Ralf Heimburger, Rudi Kramer, und Fank Spaeing. 2016. «Datenschutz geht zur Schule. Sensibler Umang mit persönlichen Daten». Arbeitsblätter. https://www.bvdnet.de/ wp-content/uploads/2016/12/Lehrerhandout_DSgzS_klicksafe.pdf.

Fischer, Claudia, Jessica Wawrzyniak, und Leena Simon. 2020. «BigBrotherAward 2020 in der Kategorie Digitalisierung». Big Brother Awards (website). https://bigbrotherawards. de/2020/digitalisierung-bildungsministerin-baden-wuerttemberg-susanne-eisenmann.

Gerick, Julia, und Birgit Eickelmann. 2019b. «Ergebnisse der wissenschaftlichen Begleitung der Evaluation des Projekts (Lernen mit digitalen Medien) (Phase II) in Schleswig-Holstein». Universität Hamburg, Universität Paderborn. https://www.schleswig-holstein.de/ DE/Fachinhalte/D/digitalesLernen/Downloads/Abschlussbericht_Evaluation.pdf;jsessioni d=EFEEB21E3876CAC44A6B97F2A3E8E538.delivery2-master?__blob=publicationFile\&v=1.

Gerick, Julia, Birgit Eickelmann, und Emilie Steglich. 2019a. "Abschlussbericht zur prozessbegleitenden Evaluation der Einführung von LOGINEO NRW an Pilotschulen». Universität Hamburg, Universität Paderborn. https://kw.uni-paderborn.de/fileadmin/fakultaet/Institute/erziehungswissenschaft/Schulpaedagogik/PDF/190706_Abschlussbericht_LNRW_FINAL.pdf.

Harvey, Lee, und Diana Green. 2000. «Qualität definieren. Fünf unterschiedliche Ansätze». In Qualität und Qualitätssicherung im Bildungsbereich; Schule, Sozialpädagogik, Hochschule, herausgegeben von Andreas Helmke, Walter Hornstein, und Ewald Terhart, 17-39. Weinheim: Beltz.

HBDI, Der Hessische Beauftragte für Datenschutz und Informationsfreiheit Hochschulen und Schulen. 2021. Duldung des HBDI für die Nutzung insbesondere US-amerikanischer Anwendungen läuft aus. https://datenschutz.hessen.de/datenschutz/hochschulen-schulen-undarchive/duldung-des-hbdi-für-die-nutzung-insbesondere-us.

Herzig, Bardo. 2020. «Digitalisierung, Medienbildung und Medienkompetenz. Verhältnisbestimmungen und Implikationen für die Lehrerbildung und den Lehrerberuf». In Digital?!: Perspektiven der Digitalisierung für den Lehrerberuf und die Lehrerbildung, herausgegeben von Martin Rothland und Simone Herrlinger, 35-50. Münster, New York: Waxmann.

Huwer, Johannes, Thomas Irion, Sebastian Kuntze, Steffen Schaal, und Christoph Thyssen. 2019. «Von TPaCK zu DPaCK: Digitalisierung im Unterricht erfordert mehr als technisches Wissen». MNU Journal, Nr. 5: 358-64.

Jude, Nina, Jeanette Ziehm, Frank Goldhammer, Hendrik Drachsler, und Marcus Hasselhorn. 2020. Digitalisierung an Schulen - eine Bestandsaufnahme. Frankfurt am Main: DIPF | Leibniz-Institut für Bildungsforschung und Bildungsinformation. https://doi. org/10.25656/01:20522. 
Kammerl, Rudolf, Alexander Unger, Silke Günther, und Anja Schwedler. 2016. «BYOD. Start in die nächste Generation. Abschlussbericht der wissenschaftlichen Evaluation des Pilotprojekts». Hamburg: Universität Hamburg. https://www.medpaed.phil.fau.de/files/2017/05/ BYOD-Projektbericht.pdf.

Kattmann, Ulrich. 2007. «Didaktische Rekonstruktion - eine praktische Theorie». In Theorien in der biologiedidaktischen Forschung: ein Handbuch für Lehramtsstudenten und Doktoranden, herausgegeben von Dirk Krüger, 93-104. Springer-Lehrbuch. Berlin [u. a.]: Springer. https://doi.org/10.1007/978-3-540-68166-3_9.

Klose, Rebecca, und Christof Schreiber. o. J. «PriMa Podcast». http://podcast.math.uni-giessen.de/primapodcast/impressum/.

KMK, Kultusministerkonferenz. 2012. «Medienbildung in der Schule». (Beschluss der Kultusministerkonferenz vom 8. März 2012). Kultusministerkonferenz. https://www.kmk.org/fileadmin/Dateien/veroeffentlichungen_beschluesse/2012/2012_03_08_Medienbildung.pdf.

KMK, Kultusministerkonferenz. 2017. «Bildung in der digitalen Welt. Strategie der Kultusministerkonferenz». https://www.kmk.org/fileadmin/pdf/PresseUndAktuelles/2018/Digitalstrategie_2017_mit_Weiterbildung.pdf.

Kosubski, Ilaria. 2021. Personalentwicklung zum Mobilen Lehren und Lernen mit Tablets. Entwicklung und Evaluation von Konzepten im Modus entwicklungsorientierter Bildungsforschung. Unveröffentlichter Masterarbeit. Goethe Universität Frankfurt.

Lorenz, Ramona, Wilfried Bos, Manuela Endberg, Birgit Eickelmann, Silke Grafe, und Jan Vahrenhold, Hrsg. 2017. Schule digital. Der Länderindikator 2017. Schulische Medienbildung in der Sekundarstufe I mit besonderem Fokus auf MINT-Fächer im Bundesländervergleich und Trends von 2015 bis 2017. Münster, New York: Waxmann. https://doi. org/10.25656/01:15656.

Moser, Heinz. 2010. Schule 2.0: Medienkompetenz für den Unterricht. 1. Aufl. Bd. 20. Schulmanagement konkret. Kronach: Link.

Müller, Antje. 2019. Aufbruch in offene Netze - Souveräne Nutzung digitaler Medien im institutionellen E-Learning. Eine rekonstruktive Fallstudie im Modus entwicklungsorientierter Bildungsforschung. PhD Thesis, Darmstadt: TU Darmstadt. http://nbn-resolving.de/ urn:nbn:de:tuda-tuprints-86316.

Müller, Jane, Mareike Thumel, Katrin Potzel, und Rudolf Kammerl. 2020. «Digital Sovereignty of Adolescents». MedienJournal 44 (1):30-40. https://doi.org/10.24989/medienjournal. v44i1.1926.

Niesyto, Horst. 2014. «Medienpädagogische Praxisforschung». In Jahrbuch Medienpädagogik 10: Methodologie und Methoden medienpädagogischer Forschung, herausgegeben von Anja Hartung, Bernd Schorb, Horst Niesyto, Heinz Moser, und Petra Grell, 173-91. Wiesbaden: Springer Fachmedien Wiesbaden. https://doi.org/10.1007/978-3-658-04718-4_9.

Petko, Dominik. 2011. «Praxisorientierte medienpädagogische Forschung: Ansätze für einen empirischen Perspektivenwechsel und eine stärkere Konvergenz von Medienpädagogik und Mediendidaktik». MedienPädagogik: Zeitschrift für Theorie und Praxis der Medienbildung 20 (0): 245-58. https://doi.org/10.21240/mpaed/20/2011.09.22.X. 
Pressestelle Hessische Staatskanzlei. 2020. «Wir müssen die Ausbreitung der Infektionen verlangsamen». https://www.hessen.de/presse/pressemitteilung/wir-muessen-die-ausbreitung-der-infektionen-verlangsamen.

Preußler, Annabell, Michael Kerres, und Mandy Schiefner-Rohs. 2014. "Gestaltungsorientierung in der Mediendidaktik: Methodologische Implikationen und Perspektiven». In Jahrbuch Medienpädagogik 10: Methodologie und Methoden medienpädagogischer Forschung, herausgegeben von Anja Hartung, Bernd Schorb, Horst Niesyto, Heinz Moser, und Petra Grell, 253-74. Wiesbaden: Springer Fachmedien Wiesbaden. https://doi.org/10.1007/9783-658-04718-4_9.

Puentedura, Ruben R. 2006. «Transformation, Technology, and Education». Maine. http:// www.hippasus.com/resources/tte/.

Rau, Franco. 2020. «Methodologische und Methodische Vorüberlegungen». MedienPädagogik: Zeitschrift für Theorie Und Praxis Der Medienbildung (Lernsituationen Metaphern Wikibooks):21-124. https://doi.org/10.21240/mpaed/diss.fr/2020.07.02.X.

Rau, Franco, Petra Grell, Anna Geritan, Britta Galanamatis, und Lars Gerber. 2021. «Bildung in der digitalen Welt - Darmstädter Modellschulen. Zwischenbericht zur Begleitung von drei Modellschulen unter Pandemiebedingungen». TU Darmstadt. https://doi.org/10.26083/tuprints-00017655.

Reber, Karin und Kaiser-Mantel, Hildegard. 2020. Apps für Schule und Therapie. Sonderpädagogik - Inklusion - Förderschwerpunkt Sprache - Sprachtherapie. http://karinreber2.paedalogis.com/fobis/AppsSchuleTherapie_Reber-KaiserMantel.pdf.

Reber, Karin, und Elisabeth Wildegger-Lack. 2020. Sprachförderung mit Medien: Von real bis digital. Wissenswertes für Eltern, Pädagogen und Therapeuten. Idstein: Schulz-Kirchner Verlag.

Reinmann, Gabi, und Werner Sesink. 2014. «Begründungslinien für eine entwicklungsorientierte Bildungsforschung». In Jahrbuch Medienpädagogik 10. Methodologie und Methoden medienpädagogischer Forschung, herausgegeben von Anja Hartung, Bernd Schorb, Horst Niesyto, Heinz Moser, und Petra Grell, 10:75-89. Wiesbaden: Springer VS. https://doi. org/10.1007/978-3-658-04718-4_4.

Rolff, Hans-Günter. 2016. Schulentwicklung kompakt: Modelle, Instrumente, Perspektiven. 3. vollständig überarbeitete und erweiterte Auflage. Weinheim: Beltz.

Schmid, Mirjam, und Dominik Petko. 2020. «Technological Pedagogical Content Knowledge» als Leitmodell medienpädagogischer Kompetenz». Herausgegeben von Klaus Rummler, Ilka Koppel, Sandra Aßmann, Patrick Bettinger, und Karsten D. Wolf. MedienPädagogik: Zeitschrift für Theorie und Praxis der Medienbildung, Jahrbuch Medienpädagogik, 17 (Jahrbuch Medienpädagogik): 121-40. https://doi.org/10.21240/mpaed/jb17/2020.04.28.X.

Schulamt Darmstadt. 2020. Rundverfügung. Nr. 07/2020 40 IV. 19.11.2020.

Schulz-Zander, Renate. 2001. «Neue Medien als Bestandteil von Schulentwicklung». In Jahrbuch Medienpädagogik 1, herausgegeben von Stefan Aufenanger, Renate Schulz-Zander, und Dieter Spanhel, 263-81. Wiesbaden: VS Verlag für Sozialwissenschaften. https://doi. org/10.1007/978-3-322-97494-5_17. 
Sesink, Werner, und Gabi Reinmann. 2015. «Umrisse eines Strukturmodells für entwicklungsorientierte bildungswissenschaftliche Forschung». In Entwicklungsorientierte Bildungsforschung. Plädoyer für einen dritten Weg in pädagogischer Forschung. Eine Textsammlung, herausgegeben von Werner Sesink, 69-83. http://www.sesink.de/wordpress/wp-content/ uploads/2015/11/Entwicklungsorientierte-Bildungsforschung_Sesink_2015.pdf.

Spanhel, Dieter. 2007. «Zur Standortbestimmung der Medienpädagogik aus anthropologischer und bildungswissenschaftlicher Sicht». In Jahrbuch Medien-Pädagogik 6: Medienpädagogik - Standortbestimmung einer erziehungswissenschaftlichen Disziplin, herausgegeben von Werner Sesink, Michael Kerres, und Heinz Moser, 33-54. Wiesbaden: VS Verlag für Sozialwissenschaften. https://doi.org/10.1007/978-3-531-90544-0_2.

Thumel, Mareike; Kammerl, Rudolf.; Irion, Thomas, Hrsg. 2020. Digitale Bildung im Grundschulalter Grundsatzfragen zum Primat des Pädagogischen. München: kopaed.

Tillmann, Alexander, und Ingo Antony. 2018. Tablet-Klassen. Begleituntersuchung, Unterrichtskonzepte und Erfahrungen aus dem Pilotprojekt «Mobiles Lernen in Hessen - MOLE». Münster; New York; München; Berlin: Waxmann. http://nbn-resolving.de/urn:nbn:de:0111pedocs-165621.

Tulodziecki, Gerhard, Silke Grafe, und Bardo Herzig. 2013. Gestaltungsorientierte Bildungsforschung und Didaktik: Theorie - Empirie - Praxis. Bad Heilbrunn: Klinkhardt.

Tulodziecki, Gerhard, Silke Grafe, und Bardo Herzig. 2014. «Praxis- und theorieorientierte Entwicklung und Evaluation von Konzepten für medienpädagogisches Handeln als gestaltungsorientierte Bildungsforschung». In Jahrbuch Medienpädagogik 10. Methodologie und Methoden medienpädagogischer Forschung, herausgegeben von Anja Hartung, Bernd Schorb, Horst Niesyto, Heinz Moser, und Petra Grell, 10:213-29. Wiesbaden: Springer VS. https://doi.org/10.1007/978-3-658-04718-4_11.

Zylka, Johannes. 2018. Digitale Schulentwicklung - Das Praxisbuch für Schulleitung und Steuergruppen. Weinheim: BELTZ. 